\title{
Preliminary report of a national survey
}

\section{The Medical Care Activities of Local Health Units}

\author{
BEVERLEE A. MYERS, M.P.H., BRUCE J. STEINHARDT, M.P.H., \\ MARY L. MOSLEY, A.B., and JOHN W. CASHMAN, M.D.
}

\section{Medicare's Effects on Medical Care}

$\mathrm{I}^{\mathrm{N}}$

$\mathrm{N}$ THE past 30 years medical care problems have become, of necessity, an active concern of many local health departments. Medical advances, changes in the prevalence of diseases, and a growing awareness of gaps in the availability and financing of medical services have led to a reshaping of the role of the local health unit in medical care activities. These changes in thinking are reflected in the 1950 and 1963 policy statements of the American Public Health Association $(1,2)$.

Despite the fact that numerous new programs and activities have been described and discussed in the literature, no comprehensive survey has been made of local health units' activities in medical care since the late forties, when Terris

Mrs. Myers is special assistant to the chief and Mr. Steinhardt is health services officer, Health Services Organization Branch, Division of Medical Care Administration, Health Services and Mental Health Administration, Public Health Service. Dr. Cashman, Assistant Surgeon General, is director of the division. Mrs. Mosley is education program specialist, Technical Operations Branch, Division of School Assistance in Federally Affected Areas, Office of Education. and Kramer reported on the national survey they performed for the Subcommittee on Medical Care of the American Public Health Association (3). In 1964, the Public Health Service undertook a nationwide survey to gather new data to use for current planning purposes, as well as to establish a baseline for future studies. The preliminary findings of this survey are reported here. Subsequent reports will deal with the findings in depth.

\section{Methods}

A nine-page questionnaire was developed and pretested to elicit information about the following: $(a)$ the characteristics of the health unit and its jurisdiction, $(b)$ its responsibilities with respect to health facilities in its jurisdiction, $(c)$ the health services it provides, $(d)$ the assistance it gives to other providers of services, $(e)$ its activities in assuring or improving the quality of services in its jurisdiction, and $(f)$ its relationships with health agencies, associations, and institutions. The study was endorsed and assisted by the Association of State and Territorial Health Officers.

In January 1966 the questionnaires were mailed to 1,703 local health units (4). In addition to routine followup of nonrespondents, special efforts were directed at units in the States which had response rates of less than 50 percent. 
Completed questionnaires were received from 1,340 units, a response rate of 78.1 percent. For a variety of reasons, 17 returned questionnaires had to be discarded.

\section{Results}

The results of this study probably do not reflect the true picture, because the units with more medical care activities likely had a higher response rate. Further, the study was not intended to be a complete inventory of all medical care activities of local health units, but rather a general overview of major activities. The response rate by State ranged from 25 to 100 percent and by region from 64 to 92 percent.

Table 1 shows the respondents by type of of health unit, population of jurisdiction, and employment status of the health officer.

\section{Table 1. Responses of health units to selected items, 1966 questionnaire survey}

\begin{tabular}{|c|c|c|c|}
\hline \multirow{2}{*}{ Item } & \multirow{2}{*}{$\begin{array}{l}\text { Number } \\
\text { question- } \\
\text { naires } \\
\text { sent }\end{array}$} & \multicolumn{2}{|c|}{ Respondents } \\
\hline & & Number & Percent \\
\hline Total & 1,703 & 1,323 & 77. 7 \\
\hline \multicolumn{4}{|l|}{ Type of health unit: ${ }^{1}$} \\
\hline County & 852 & 687 & 80. \\
\hline City & 344 & 247 & 71. \\
\hline City-county & 90 & 74 & 82. \\
\hline State health district & 182 & 123 & 67. \\
\hline Local health district_ & 235 & 192 & 81. 7 \\
\hline $\begin{array}{l}\text { Population of } \\
\text { jurisdiction: }{ }^{2}\end{array}$ & & & \\
\hline Under 15,000 & 215 & 170 & 79. \\
\hline $15,000-24,999 \ldots$ & 238 & 202 & $8=$ \\
\hline $25,000-44,999 \ldots$ & 352 & 266 & 75. \\
\hline $45,000-74,999$ & 309 & 244 & 79. \\
\hline $75,000-199,999 \ldots$ & 277 & 255 & 81. 2 \\
\hline 200,000 and over..... & 187 & 164 & 87. 7 \\
\hline \multirow{2}{*}{\multicolumn{4}{|c|}{$\begin{array}{c}\text { Employment status of } \\
\text { health officer: }{ }^{3}\end{array}$}} \\
\hline & & & \\
\hline Full time. & 950 & 763 & 80. \\
\hline Part time $\ldots \ldots$ & 580 & 442 & 76. \\
\hline No health officer & 140 & 94 & 67. 1 \\
\hline Unknown_........... & 33 & 24 & 72.7 \\
\hline
\end{tabular}

1 Type is generally that listed in the "Directory of Local Health Units, 1964," compiled by the Public Health Service. This information was updated by the office which compiled the directory.

${ }^{2}$ Figures for respondents are those reported on questionnaires. Figures for nonrespondents are from 1960 census data. Unknowns include localities not reported by the census; for example, those in Puerto Rico.

3 Figures for respondents are those reported on questionnaires. Figures for nonrespondents are from the proofs of the "Directory of Local Health Units, 1966."
Tables 2-4 give an overview of some of the characteristics of the responding local health units, including regional location, type of unit, status and degrees of the health officer, and expenditures.

Slightly more than half of the units were classified as county health departments, but the proportion of different types of units by region was quite variable (table 2 ). Thus, city health departments predominate in the populous New England States, while in the southern and western Mountain States two-thirds or more of the units are county health departments or local health districts.

Eighty percent of the local health units were directed by a physician health officer, and almost a third of these physicians had a public health degree (table 3 ). However, only 58 percent of the responding units reported having a full-time health officer.

Of 1,010 health units for which it was possible to calculate per capita public health expenditures, approximately 60 percent reported that these expenditures were less than $\$ 2$ (table 4). While there is great variability regionally and by type of health unit, units serving very small jurisdictions and units serving very large jurisdictions tend to have above average per capita public health expenditures.

Tables 5-7 show general findings about the kinds of health facilities and programs available in the units' jurisdictions and the kinds of responsibilities the local health unit assumes in relation to each.

The responsibilities which the health units most frequently assumed for available health facilities were inspection, licensure, and operation (table 5). The facilities most frequently operated by local health units were mental health clinics and clinical laboratories; however, inspection and licensing of these facilities by local health units were infrequent. Inspection without licensing was the most frequent responsibility assumed with respect to general and special hospitals and skilled nursing homes in their jurisdictions, although almost 30 percent of the health units licensed skilled nursing homes.

Of the 10 selected health services listed in tables 6 and 7 , the two most prevalent were school health (95 percent) and crippled children's (94.1 percent). The health unit was the 
Table 2. Type of health unit, by Public Health Service Region

\begin{tabular}{|c|c|c|c|c|c|c|c|c|c|c|c|}
\hline \multirow{2}{*}{ Region ${ }^{1}$} & \multirow{2}{*}{$\begin{array}{c}\text { Total } \\
\text { re- } \\
\text { spond- } \\
\text { dents }\end{array}$} & \multicolumn{2}{|c|}{ County } & \multicolumn{2}{|c|}{ City } & \multicolumn{2}{|c|}{ City-county } & \multicolumn{2}{|c|}{$\begin{array}{l}\text { State health } \\
\text { district }\end{array}$} & \multicolumn{2}{|c|}{$\begin{array}{c}\text { Local health } \\
\text { district }\end{array}$} \\
\hline & & $\underset{\text { ber }}{\text { Num- }}$ & $\begin{array}{l}\text { Per- } \\
\text { cent }\end{array}$ & $\underset{\text { ber }}{\text { Num- }}$ & $\begin{array}{l}\text { Per- } \\
\text { cent }\end{array}$ & $\underset{\text { ber }}{\text { Num- }}$ & $\begin{array}{l}\text { Per- } \\
\text { cent }\end{array}$ & $\underset{\text { ber }}{\text { Num- }}$ & $\begin{array}{l}\text { Per- } \\
\text { cent }\end{array}$ & $\underset{\text { ber }}{\text { Num- }}$ & $\begin{array}{l}\text { Per- } \\
\text { cent }\end{array}$ \\
\hline Total & 1,323 & 687 & 51. 9 & 247 & 18. 7 & 74 & 5.6 & 123 & 9. 3 & 192 & 14.5 \\
\hline I. & 90 & 1 & 1.1 & 77 & 85.6 & 0 & 0 & 10 & 11.1 & 2 & 2.2 \\
\hline & 98 & 24 & 24. 5 & 56 & 57. 1 & 0 & 0 & 16 & 16. 3 & ? & 2. 0 \\
\hline & 310 & $\begin{array}{r}189 \\
\end{array}$ & 61. 0 & 12 & 3. 9 & 17 & 5. 5 & 58 & 18. 7 & 34 & 11. 0 \\
\hline & 223 & 131 & 58. 7 & 4 & 1. 8 & 2 & .9 & 0 & 0 & 86 & 38.6 \\
\hline & 207 & 95 & 45. 9 & 68 & 32.9 & 3 & 1. 4 & 6 & 2. 9 & 35 & 16. 9 \\
\hline & 95 & $\begin{array}{l}95 \\
45\end{array}$ & $\begin{array}{l}47.9 \\
47.4\end{array}$ & $\begin{array}{l}00 \\
16\end{array}$ & 16. 8 & 15 & $\begin{array}{l}1.4 \\
15.8\end{array}$ & 14 & 14.7 & $\begin{array}{r}50 \\
5\end{array}$ & 5. 3 \\
\hline VII & 170 & 124 & 72.9 & 4 & 24.0 & 23 & 13.5 & 10 & 5.9 & 9 & 5. 3 \\
\hline VIIII-- & 22 & 7 & 31.8 & 1 & 4. 5 & 6 & 27. 3 & 0 & 0 & 8 & 36.4 \\
\hline IX & 108 & 71 & 65. 7 & 9 & 8. 3 & 8 & 7. 4 & 9 & 8.3 & 11 & 10. 2 \\
\hline
\end{tabular}

${ }^{1}$ States in each region are: I, Connecticut, Maine, Massachusetts, New Hampshire, Rhode Island, and Vermont; II, Delaware, New Jersey, New York, and Pennsylvania; III, District of Columbia, Kentucky, Maryland, North Carolina, Puerto Rico, Virginia, Virgin Islands, and West Virginia ; IV, Alabama, Florida, Georgia, Mississippi, South Carolina, and Tennessee;
V, Illinois, Indiana, Michigan, Ohio, and Wisconsin; VI, Iowa, Kansas, Minnesota, Missouri, Nebraska, North Dakota, and South Dakota ; VII, Arkansas, Louisiana, New Mexico, Oklahoma, and Texas; VIII, Colorado, Idaho, Montana, Utah, and Wyoming ; IX, Alaska, Arizona, California, Hawaii, Nevada, Oregon, and Washington.

Table 3. Employment status and academic degrees of health officers in 1,323 local health units

Health officers $\frac{\text { Full time }}{\text { Number Percent }} \frac{\text { Part time }}{\text { Number Percent }} \frac{\text { No health officer }}{\text { Number Percent }} \frac{\text { Unknown }}{\text { Number Percent }}$

\begin{tabular}{|c|c|c|c|c|c|c|c|c|}
\hline M.D.'s with public health & 333 & 43.7 & 79 & 17.8 & 0 & 0 & 3 & 12. 0 \\
\hline M.D.'s without public health & 297 & 39.0 & 337 & 76.4 & 0 & 0 & 11 & 44.0 \\
\hline $\begin{array}{l}\text { Non-M.D.'s with public health } \\
\text { degree.- } \\
\text { Non-M.D.'s without public }\end{array}$ & 25 & 3. 3 & 1 & .2 & 0 & 0 & 0 & 0 \\
\hline $\begin{array}{l}\text { health degree. } \\
\text { None } \\
\text { Unknown }\end{array}$ & $\begin{array}{r}91 \\
0 \\
16\end{array}$ & $\begin{array}{l}11.9 \\
0 \\
2.1\end{array}$ & $\begin{array}{r}15 \\
0 \\
9\end{array}$ & $\begin{array}{l}3.5 \\
0 \\
2.0\end{array}$ & $\begin{array}{r}0 \\
95 \\
0\end{array}$ & $\begin{array}{c}0 \\
100.0 \\
0\end{array}$ & $\begin{array}{r}1 \\
0 \\
10\end{array}$ & $\begin{array}{rl}4.0 & 0 \\
40.0 & \end{array}$ \\
\hline Total & 762 & 100. 0 & 441 & 100.0 & 95 & 100.0 & 25 & 00.0 \\
\hline
\end{tabular}

Table 4. Per capita public health expenditures of 1,323 local health units, by population of jurisdiction

\begin{tabular}{|c|c|c|c|c|c|c|c|c|c|c|c|c|c|c|c|}
\hline \multirow{2}{*}{ Population of jurisdiction } & \multirow{2}{*}{$\begin{array}{l}\text { Total } \\
\text { health } \\
\text { units }\end{array}$} & \multicolumn{2}{|c|}{ Under $\$ 1$} & \multicolumn{2}{|c|}{$\$ 1-\$ 1.49$} & \multicolumn{2}{|c|}{$\$ 1.50-\$ 1.99$} & \multicolumn{2}{|c|}{$\$ 2-\$ 2.99$} & \multicolumn{2}{|c|}{$\$ 3-\$ 3.99$} & \multicolumn{2}{|c|}{$\$ 4$ and over } & \multicolumn{2}{|c|}{ Unknown } \\
\hline & & $\begin{array}{c}\text { Num- } \\
\text { ber }\end{array}$ & $\begin{array}{l}\text { Per- } \\
\text { cent }\end{array}$ & $\begin{array}{c}\text { Num- } \\
\text { ber }\end{array}$ & $\begin{array}{l}\text { Per- } \\
\text { cent }\end{array}$ & $\begin{array}{l}\text { Num- } \\
\text { ber }\end{array}$ & $\begin{array}{l}\text { Per- } \\
\text { cent }\end{array}$ & $\begin{array}{l}\text { Num- } \\
\text { ber }\end{array}$ & $\begin{array}{l}\text { Per- } \\
\text { cent }\end{array}$ & $\begin{array}{l}\text { Num- } \\
\text { ber }\end{array}$ & $\begin{array}{l}\text { Per- } \\
\text { cent }\end{array}$ & $\begin{array}{l}\text { Num- } \\
\text { ber }\end{array}$ & $\begin{array}{l}\text { Per- } \\
\text { cent }\end{array}$ & $\begin{array}{l}\text { Num- } \\
\text { ber }\end{array}$ & $\begin{array}{l}\text { Per- } \\
\text { cent }\end{array}$ \\
\hline Total . & 1,323 & 162 & 12.2 & 230 & 17.4 & 211 & 15.9 & 232 & 17.5 & 86 & 6.5 & 89 & 6. 7 & 313 & 23.7 \\
\hline Under $15,000 \ldots \ldots$ & 170 & 17 & 10.0 & 21 & 12.4 & 29 & 17.1 & 29 & 17. 1 & 13 & 7.6 & 18 & 10.6 & 43 & 25.3 \\
\hline $15,000-24,999 \ldots$ & 202 & 31 & 15.3 & 52 & 25.7 & 28 & 13.9 & 26 & 12.9 & 9 & 4.5 & 19 & 9.4 & 37 & 18. 3 \\
\hline $25,000-44,999 \ldots \ldots$ & 266 & 39 & 14. 7 & 56 & 21.1 & 43 & 16. 2 & 51 & 19.2 & 11 & 4.1 & 12 & 4.5 & 54 & 20.3 \\
\hline $45,000-74,999 \ldots \ldots$ & 244 & 33 & 13.5 & 48 & 19.7 & 43 & 17.6 & 36 & 14.8 & 18 & 7.4 & 14 & 5.7 & 52 & 21.3 \\
\hline $75,000-199,999 \ldots \ldots \ldots \ldots$ & 225 & 23 & 10.2 & 33 & 14. 7 & 46 & 20.4 & 48 & 21.3 & 20 & 8.9 & 11 & 4.9 & 44 & 19.6 \\
\hline 200,000 and over & 164 & 19 & 11.6 & 20 & 12.2 & 22 & 13.4 & 42 & 25.6 & 15 & 9.1 & 15 & 9.1 & 31 & 18. 9 \\
\hline Unknown & 52 & 0 & 0 & 0 & 0 & 0 & 0 & 0 & 0 & 0 & 0 & 0 & 0 & 52 & 100.0 \\
\hline
\end{tabular}


sole operator of the school health program in more than half of the responding jurisdictions. In 51.2 percent of the jurisdictions where it was available, the crippled children's program was operated by both the health unit and another agency.
The responses in relation to homemaker services and vocational rehabilitation are particularly interesting, because in more than 80 percent of the jurisdictions where these programs were available they were operated by both the health unit and another agency.

Table 5. Responsibilities assumed by 1,323 local health units for each type of health facility available in their jurisdictions

\begin{tabular}{|c|c|c|c|c|c|c|c|c|c|c|c|c|c|c|}
\hline \multirow{3}{*}{ Type of health facility } & \multirow{2}{*}{\multicolumn{2}{|c|}{$\begin{array}{l}\text { Jurisdictions } \\
\text { with facility }\end{array}$}} & \multicolumn{12}{|c|}{ Responsibilty of local health unit } \\
\hline & & & \multicolumn{2}{|c|}{$\begin{array}{l}\text { No respon- } \\
\text { sibility }\end{array}$} & \multicolumn{2}{|c|}{ Operation } & \multicolumn{2}{|c|}{ License only } & \multicolumn{2}{|c|}{ Inspect only } & \multicolumn{2}{|c|}{$\begin{array}{l}\text { License and } \\
\text { inspect }\end{array}$} & \multicolumn{2}{|c|}{ Other 1} \\
\hline & Num- & $\begin{array}{l}\text { Per- } \\
\text { cent }\end{array}$ & Num- & $\begin{array}{l}\text { Per- } \\
\text { cent }\end{array}$ & Num- & $\begin{array}{l}\text { Per- } \\
\text { cent }\end{array}$ & Num- & $\begin{array}{l}\text { Per- } \\
\text { cent }\end{array}$ & Num- & $\begin{array}{l}\text { Per- } \\
\text { cent }\end{array}$ & Num- & $\begin{array}{l}\text { Per- } \\
\text { cent }\end{array}$ & Num- & $\begin{array}{l}\text { Per- } \\
\text { cent }\end{array}$ \\
\hline General hospital........ & 1,054 & 79.7 & 493 & 46.8 & 54 & 5.1 & 149 & 14.1 & 326 & 30.9 & 18 & 1.7 & 79 & 7.5 \\
\hline Special hospital.......... & 353 & 26.7 & 175 & 49.6 & 16 & 4.5 & 29 & 8.2 & 101 & 28.6 & 4 & 1.1 & 47 & 13.3 \\
\hline 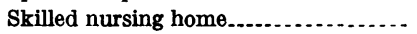 & 913 & 69.0 & 229 & 25.1 & 68 & 7.4 & 272 & 29.8 & 331 & 36. 3 & 36 & 3.9 & 91 & 10.0 \\
\hline Rehabilitation center & 298 & 22.5 & 202 & 67.8 & 12 & 4. 0 & 15 & 5.0 & 36 & 12.1 & 1 & .3 & 40 & 13.4 \\
\hline Mental health clinic & 630 & 47. 6 & 375 & 59.5 & 144 & 22.9 & 14 & 2.2 & 13 & 2.1 & 0 & 0 & 98 & 15.6 \\
\hline Clinical laboratory & 568 & 42.9 & 387 & 68.1 & 118 & 20.8 & 22 & 3.9 & 21 & 3.7 & 1 & .2 & 37 & 6.5 \\
\hline
\end{tabular}

1 Other responsibilities most frequently included consultation and advice, referrals, and followup with public health nursing.

Table 6. Programs available in jurisdictions of 1,323 local health units, by agency operating program

\begin{tabular}{|c|c|c|c|c|c|c|c|c|c|c|}
\hline \multirow{3}{*}{ Program } & \multirow{2}{*}{\multicolumn{2}{|c|}{$\begin{array}{l}\text { Jurisdictions with } \\
\text { organized programs }\end{array}$}} & \multicolumn{8}{|c|}{ Agency operating program } \\
\hline & & & \multicolumn{2}{|c|}{ Health unit only } & \multicolumn{2}{|c|}{ Other agency only } & \multicolumn{2}{|c|}{$\begin{array}{c}\text { Health unit and } \\
\text { other agency }\end{array}$} & \multicolumn{2}{|c|}{ Unknown } \\
\hline & Number & Percent & Number & Percent & Number & Percent & Number & Percent & Number & Percent \\
\hline Information and referral service............ & 996 & 75.3 & 353 & 35.4 & 463 & 46.5 & 144 & 14.5 & 36 & 3.6 \\
\hline Home nusing service... & 486 & 36.7 & 192 & 39.5 & 51 & 10.5 & 217 & 44.7 & 26 & 5.3 \\
\hline Homemaker service......... & 310 & 23.4 & 25 & 8.1 & 7 & 2.3 & 250 & 80.6 & 28 & 9.0 \\
\hline Home health service & 628 & 47.5 & 349 & 55.6 & 96 & 15.3 & 141 & 22.5 & 42 & 6.7 \\
\hline Medical care for recipients of public assistance..... & 1,082 & 81.8 & 123 & 11.4 & 122 & 11.3 & 780 & 72.1 & 57 & 5.3 \\
\hline Crippled children $\ldots . . . . . . . . . . . . . .$. & 1,245 & 94.1 & 304 & 24.4 & 248 & 19.9 & 637 & 51.2 & 56 & 4.5 \\
\hline School health & 1,257 & 95.0 & 671 & 53.4 & 317 & 25.2 & 204 & 16.2 & 65 & 5.2 \\
\hline Vocational rehabilitation & 1,087 & 82.2 & 21 & 1.9 & 48 & 4.4 & 957 & 88.0 & 61 & 5.6 \\
\hline 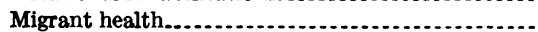 & 299 & 22.6 & 147 & 49.2 & 60 & 20.1 & 76 & 25.4 & 16 & 5.4 \\
\hline Community mental health & 768 & 58.0 & 108 & 14.1 & 77 & 10.0 & 516 & 67.2 & 67 & 8.7 \\
\hline
\end{tabular}

Table 7. Type of assistance given to agencies operating medical care programs in jurisdictions of 1,323 local health units

\begin{tabular}{|c|c|c|c|c|c|c|c|c|c|c|c|}
\hline \multirow{3}{*}{ Program } & \multirow{3}{*}{$\begin{array}{c}\text { Number of } \\
\text { jurisdictions } \\
\text { with } \\
\text { organized } \\
\text { programs }\end{array}$} & \multirow{2}{*}{\multicolumn{2}{|c|}{$\begin{array}{l}\text { Jurisdictions } \\
\text { where other } \\
\text { agency operates } \\
\text { service 1 }\end{array}$}} & \multicolumn{8}{|c|}{ Assistance health unit provides to other agency } \\
\hline & & & & \multicolumn{2}{|c|}{$\begin{array}{l}\text { Consultation } \\
\text { and advice }\end{array}$} & \multicolumn{2}{|c|}{$\begin{array}{c}\text { Services or } \\
\text { personnel or both }\end{array}$} & \multicolumn{2}{|c|}{ Other ${ }^{2}$} & \multicolumn{2}{|c|}{ No assistance } \\
\hline & & Number & Percent & Number & Percent & Number & Percent & Number & Percent & Number & Percent \\
\hline Information and referral service...... & 996 & 607 & 60.9 & 520 & 85.7 & 320 & 52.7 & 23 & 3.8 & 53 & 8.7 \\
\hline Home nursing service & 486 & 268 & 55.1 & 169 & 63.1 & 74 & 27.6 & 30 & 11.2 & 58 & 21.6 \\
\hline Homemaker service.................... & 310 & 257 & 82.9 & 125 & 48.6 & 34 & 13.2 & 17 & 6.6 & 107 & 41.6 \\
\hline Home health service. . ................ & 628 & 237 & 37.7 & 147 & 62.0 & 90 & 38.0 & 23 & 9.7 & 59 & 24.9 \\
\hline Medical care for recipients of public & & & & & & & & & & & \\
\hline assistance... & 1,082 & 902 & 83.4 & 530 & 58.8 & 313 & 34.7 & 36 & 4.0 & 274 & 30.4 \\
\hline Crippled children & 1,245 & 885 & 71.1 & 529 & 59.8 & 493 & 55.7 & 65 & 7.3 & 164 & 18.5 \\
\hline School health.......................... & 1,257 & 521 & 41.4 & 428 & 82.1 & 343 & 65.8 & 18 & 3.5 & 53 & 10.2 \\
\hline Vocational rehabilitation.............. & 1,087 & 1,005 & 92.5 & 608 & 60.5 & 251 & 25.0 & 83 & 8.3 & 300 & 29.9 \\
\hline Migrant health & 299 & 136 & 45.5 & 91 & 66.9 & 71 & 52.2 & 3 & 2.2 & 31 & 22.8 \\
\hline Community mental health........... & 768 & 593 & 77.2 & 335 & 56.5 & 213 & 35.9 & 63 & 10.6 & 163 & 27.5 \\
\hline
\end{tabular}

1 Either alone or in addition to service operated by health unit.

2 Other most frequently included referrals, followup, coordination of services, and financial assistance. 
Table 8. Method of providing service in medical care programs of health units

\begin{tabular}{|c|c|c|c|c|c|c|c|c|c|c|c|}
\hline \multirow{3}{*}{$\begin{array}{l}\text { Program and services provided } \\
\text { in program }\end{array}$} & \multirow{3}{*}{$\begin{array}{l}\text { Units with } \\
\text { specified } \\
\text { program } \\
\text { or service }\end{array}$} & \multicolumn{10}{|c|}{ Method of providing service } \\
\hline & & \multicolumn{2}{|c|}{$\begin{array}{l}\text { Through own } \\
\text { staff or } \\
\text { facility only }\end{array}$} & \multicolumn{2}{|c|}{$\begin{array}{l}\text { Purchased from } \\
\text { other } \\
\text { source only }\end{array}$} & \multicolumn{2}{|c|}{$\begin{array}{l}\text { Through own } \\
\text { staff and } \\
\text { purchased }\end{array}$} & \multicolumn{2}{|c|}{ Other 1} & \multicolumn{2}{|c|}{ Unknown } \\
\hline & & Number & Percent & Number & Percent & Number & Percent & Number & Percent & Number & Percent \\
\hline Child health program. & 1,105 & & & & & & & & & & \\
\hline Well child-supervision. & 1,060 & 873 & 82.4 & 35 & 3.3 & 110 & 10.4 & 5 & 0.5 & 37 & 3.5 \\
\hline In-hospital care & 235 & 58 & 24.7 & 147 & 62.6 & 9 & 3.8 & 7 & 3.0 & 14 & 6.0 \\
\hline Physicians' services. . & 388 & 196 & 50.5 & 106 & 27.3 & 48 & 12.4 & 11 & 2.8 & 27 & 7.0 \\
\hline Drugs................. & $\mathbf{3 7 0}$ & 182 & 49.2 & 98 & 26.5 & 51 & 13.8 & 13 & 3.5 & 26 & 7.0 \\
\hline Clinical laboratory & 399 & 199 & 49.9 & 98 & 24.6 & 44 & 11.0 & 26 & 6.5 & 32 & 8.0 \\
\hline Adult health program. & 694 & & & & & & & & & & \\
\hline Health maintenance. & 604 & 433 & 71.7 & 37 & 6.1 & 69 & 11.4 & 3 & .5 & 62 & 10.3 \\
\hline In-hospital care & 187 & 55 & 29.4 & 95 & 50.8 & 10 & 5.3 & 8 & 4.3 & 19 & 10.2 \\
\hline Physicians' services... & 293 & 162 & 55.3 & 61 & 20.8 & 36 & 12.3 & 6 & 2.0 & 28 & 9.6 \\
\hline Drugs.................. & 324 & 166 & 51.2 & 71 & 21.9 & 47 & 14.5 & 12 & 3.7 & 28 & 8.6 \\
\hline Clinical laboratory.... & 348 & 182 & 52.3 & 77 & 22.1 & 38 & 10.9 & 19 & 5.5 & 32 & 9.2 \\
\hline Venereal disease program. & 1,124 & & & & & & & & & & \\
\hline Screening or diagnosis or both & 1,030 & 827 & 80.3 & 65 & 6.3 & 85 & 8.3 & 11 & 1.1 & 42 & 4. 1 \\
\hline Physicians' services................... & 883 & 665 & 75.3 & 115 & 13.0 & 67 & 7.6 & 12 & 1.4 & 24 & 2.7 \\
\hline Drugs.................. & 997 & 748 & 75.0 & 109 & 10.9 & 65 & 6.5 & 40 & 4.0 & 35 & $\mathbf{3 . 5}$ \\
\hline Clinical laboratory.... & 887 & 560 & 63.1 & 180 & 20.3 & 60 & 6.8 & 56 & 6. 3 & 31 & 3.5 \\
\hline Tuberculosis program. & 1,278 & & & & & & & & & & ....... \\
\hline Screening or diagnosis or both........ & 1,245 & 812 & 65.2 & 78 & 6.3 & 170 & 13.7 & 28 & 2.2 & 157 & 12.6 \\
\hline In-hospital care. & 689 & 150 & 21.8 & 385 & 55.9 & 30 & 4.4 & 78 & 11.3 & 46 & 6.7 \\
\hline Physicians' services.......... & 816 & 426 & 52.2 & 205 & 25.1 & 94 & 11.5 & 41 & 5.0 & 50 & 6. 1 \\
\hline 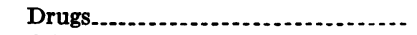 & 1,036 & $\mathbf{5 7 5}$ & 55.5 & 225 & 21.7 & 99 & 9.6 & 59 & 5.7 & 78 & 7.5 \\
\hline Clinical laboratory & 910 & 455 & 50.0 & 259 & 28.5 & 72 & 7.9 & 71 & 7.8 & 53 & 5.8 \\
\hline Maternal health progra & 856 & & & & & & & & & & \\
\hline Prenatal care......... & 822 & 657 & 79.9 & 42 & 5.1 & 61 & 7.4 & 11 & 1.3 & 51 & 6. 2 \\
\hline Drugs......... & 447 & 284 & 63.5 & 68 & 15.2 & 46 & 10.3 & 15 & 3.4 & 34 & 7.6 \\
\hline Clinical laboratory & 581 & 352 & 60.6 & 96 & 16.5 & 69 & 11.9 & 22 & 3.8 & 42 & 7.2 \\
\hline Delivery in home............. & 90 & 40 & 44.4 & 31 & 34.4 & 5 & 5.6 & 2 & 2.2 & 12 & 13.3 \\
\hline Delivery in hospital....................... & 265 & 62 & 23.4 & 152 & 57.4 & 7 & 2.6 & 13 & 4.9 & 30 & 11.3 \\
\hline Crippled children's program. & 950 & & & & & & & & & & \\
\hline Screening or diagnosis or both....... & 910 & 460 & 50.5 & 162 & 17.8 & 178 & 19.6 & 51 & 5.6 & 59 & 6. 5 \\
\hline In-hospital care & 611 & 95 & 15.5 & 400 & 65.5 & 28 & 4. 6 & 75 & 12.3 & 13 & 2.1 \\
\hline Physicians' services.................... & 659 & 160 & 24.3 & 349 & 53.0 & 66 & 10.0 & 70 & 10.6 & 14 & 2.1 \\
\hline 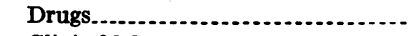 & 558 & 139 & 24.9 & 296 & 53.0 & 51 & 9.1 & 58 & 10.4 & 14 & 2.5 \\
\hline Clinical laboratory & 580 & 151 & 26.0 & 302 & 52.1 & 56 & 9.7 & 56 & 9.7 & 15 & 2.6 \\
\hline Dental health program & 869 & & & & & & & & & & \\
\hline Inspection and referral. & 788 & 592 & 75.1 & 86 & 10.9 & 61 & 7.7 & 17 & 2.2 & 32 & 4.1 \\
\hline Prophylactic servicas................. & 627 . & 438 & 69.9 & 125 & 19.9 & 35 & 5.6 & 11 & 1.8 & 18 & 2.9 \\
\hline Other treatment. & 567 & 347 & 61.2 & 154 & 27.2 & 35 & 6.2 & 10 & 1.8 & 21 & 3. 7 \\
\hline Heart disease control program. & 759 & & & & & & & & & & \\
\hline Screening or diagnosis or both...... & 581 & 308 & 53.0 & 89 & 15.3 & 101 & 17.4 & 23 & 4.0 & 60 & 10.3 \\
\hline In-hospital care & 306 & 76 & 24.8 & 184 & 60.1 & 13 & 4.2 & 21 & 6.9 & 12 & 3.9 \\
\hline Physicians' services................. & 393 & 158 & 40.2 & 154 & 39.2 & 41 & 10.4 & 18 & 4. 6 & 20 & 5. 1 \\
\hline Drugs.......... & 606 & 304 & 50.2 & 153 & 25.2 & 65 & 10.7 & 52 & 8.6 & 32 & 5.3 \\
\hline Clinical laboratory & 378 & 156 & 41.3 & 151 & 39.9 & 33 & 8.7 & 25 & 6.6 & 13 & 3.4 \\
\hline $\begin{array}{l}\text { Cancer control program } \\
\text { Screening or diagnosis or both }\end{array}$ & $\begin{array}{l}561 \\
497\end{array}$ & 268 & 53. 9 & 95 & 19.1 & 70 & 14. 1 & 23 & 4.6 & 41 & 8.2 \\
\hline In-hospital diagnosis. & 262 & 41 & 15.6 & 166 & 63.4 & 9 & 3.4 & 24 & 9.2 & 22 & 8.4 \\
\hline In-hospital care & 241 & 35 & 14.5 & 157 & 65.1 & 7 & 2.9 & 33 & 13.7 & 9 & 3. 7 \\
\hline Physicians' services............ & 244 & 70 & 28.7 & 119 & 48.8 & 20 & 8.2 & 26 & 10.7 & 9 & 3. 7 \\
\hline Drugs_....... & 223 & 43 & 19.3 & 127 & 57.0 & 17 & 7.6 & 24 & 10.8 & 12 & 5.4 \\
\hline Clinical laboratory & 270 & 66 & 24.4 & 149 & 55.2 & 21 & 7.8 & 24 & 8.9 & 10 & 3. 7 \\
\hline Mental health program.. & 730 & & & & & & & & & & \\
\hline Diagnosis.................... & 544 & 262 & 48. 2 & 152 & 27.9 & 77 & 14. 2 & 20 & 3.7 & 33 & 6. 1 \\
\hline Ambulatory care & 337 & 151 & 44. 8 & 112 & 33.2 & 36 & 10.7 & 14 & 4. 2 & 24 & 7.1 \\
\hline 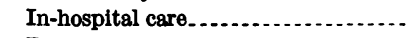 & 203 & 28 & 13.8 & 137 & 67.5 & 12 & 5.9 & 16 & 7.9 & 10 & 4. 9 \\
\hline Drugs.................. & 295 & 117 & 39.7 & 112 & 38.0 & 33 & 11.2 & 16 & 5.4 & 17 & 5.8 \\
\hline 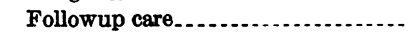 & 590 & 438 & 74. 2 & 52 & 8.8 & 47 & 8.0 & 13 & 2.2 & 40 & 6.8 \\
\hline
\end{tabular}

1 Other includes donations, State programs, and combinations of these with staff provided and purchased. 
In jurisdictions where programs were available but operated by another agency, either alone or in addition to the health unit's program, the most common form of assistance provided by the health unit was consultation and advice. In more than 85 percent of the juris- dictions where the information and referral service was operated by another agency, the local health unit provided consultation and advice. Assistance in the form of services and personnel was provided by the local health unit in more than 50 percent of the jurisdictions

Table 9. Index of involvement of 1,323 local health units in organizing medical care, by number of programs and services offered

\begin{tabular}{|c|c|c|c|c|c|c|c|c|c|c|c|c|c|}
\hline \multirow{3}{*}{ Number of programs and services offered } & \multirow{3}{*}{$\begin{array}{c}\text { Total } \\
\text { health } \\
\text { units }\end{array}$} & \multicolumn{12}{|c|}{ Index } \\
\hline & & \multicolumn{2}{|r|}{ I } & \multicolumn{2}{|c|}{ II } & \multicolumn{2}{|c|}{ III } & \multicolumn{2}{|c|}{ IV } & \multicolumn{2}{|c|}{$\mathrm{V}$} & \multicolumn{2}{|c|}{ VI } \\
\hline & & \multicolumn{2}{|c|}{$\underset{\text { ber }}{\text { Num- Percent }}$} & \multicolumn{2}{|c|}{$\underset{\text { ber }}{\text { Num- Percent }}$} & \multicolumn{2}{|c|}{$\underset{\text { ber }}{\text { Num- Percent }}$} & \multicolumn{2}{|c|}{$\underset{\text { ber }}{\text { Num- Percent }}$} & \multicolumn{2}{|c|}{$\underset{\text { ber }}{\text { Num- Percent }}$} & \multicolumn{2}{|c|}{$\underset{\text { ber }}{\text { Num- Percent }}$} \\
\hline Total $\ldots$ & 1,323 & 76 & 5.7 & 149 & 11.3 & 248 & 18.7 & 329 & 24.9 & 320 & 24.2 & 201 & 15.2 \\
\hline $\begin{array}{l}\text { No program } \\
1 \text { program: }\end{array}$ & 20 & $\ldots$ & & & & & & & & 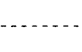 & 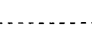 & 20 & 100.0 \\
\hline 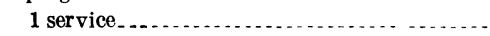 & 19 & & & & & & & & & & & 19 & 100.0 \\
\hline 2-3 services.............. & 3 & & & & & & & & & & & 3 & 100.0 \\
\hline More than 3 services.. & 4 & & & & & & & & & & ..... & 4 & 100.0 \\
\hline \multicolumn{14}{|l|}{2 programs: } \\
\hline 2 services.. & 11 & & & & & & & & & 0 & 0 & 11 & 100.0 \\
\hline 3-7 services.......... & 23 & & & & & & & & & 0 & 0 & 23 & 100.0 \\
\hline More than 7 services.. & 1 & & & & & & & $\ldots .$. & ....... & 1 & 100.0 & 0 & 0 \\
\hline 3 programs: & & & & & & & & & & & & & \\
\hline 3 services.. & 20 & & & & & & & & & 0 & 0 & 20 & 100.0 \\
\hline 4-10 services......... & 49 & & & & & & & 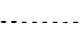 & …... & 19 & 38.8 & 30 & 61.2 \\
\hline More than 10 services... & 8 & & & & & & & & $\cdots$ & 8 & 100.0 & 0 & 0 \\
\hline 4 programs: & & & & & & & & & & & & & \\
\hline 4 services. & 6 & & & & & & & 0 & 0 & 0 & 0 & 6 & 100.0 \\
\hline 5-10 services........... & 59 & & & & & & & 0 & 0 & 28 & 47.5 & 31 & 52.5 \\
\hline 11-15 services.......... & 21 & & & & & & & 0 & 0 & 21 & 100.0 & 0 & 0 \\
\hline More than 15 services. & 8 & & & & & & ..... & 8 & 100.0 & 0 & 0 & 0 & 0 \\
\hline 5 programs: & & & & & & & & & & & & & \\
\hline 5 services..... & 5 & & & & & & & 0 & 0 & 0 & 0 & 5 & 100.0 \\
\hline 6-12 services. . . . . . . & 60 & & & & & & & 0 & 0 & 45 & 75.0 & 15 & 25.0 \\
\hline 13-20 services.......... & 63 & & & & & & & 32 & 50.8 & 31 & 49.2 & 0 & 0 \\
\hline More than 21 services. & 4 & & & & & & & 4 & 100.0 & 0 & 0 & 0 & 0 \\
\hline 6 programs: & & & & & & & & & & & & & \\
\hline 6 services...... & 9 & & & & & 0 & 0 & 0 & 0 & 0 & 0 & 9 & 100.0 \\
\hline 7-15 services... & 66 & & & & & 0 & 0 & 0 & 0 & 62 & 93.9 & 4 & 6.1 \\
\hline 16-24 services. . . . . . . & 61 & & & & & 5 & 8.2 & 56 & 91.8 & $\mathbf{0}$ & 0 & 0 & 0 \\
\hline More than 24 services. & 5 & & & & & 5 & 100.0 & 0 & 0 & 0 & 0 & 0 & 0 \\
\hline 7 programs: & & & & & & & & & & & & & \\
\hline 7 services......... & 1 & & .... & 0 & 0 & 0 & 0 & 0 & 0 & 0 & 0 & 1 & 100.0 \\
\hline 8-18 services. . . . . . & 86 & & $\ldots$ & 0 & 0 & 0 & 0 & 33 & 38.4 & 53 & 61.6 & 0 & 0 \\
\hline 19-28 services. . . . . . . & 102 & & & 0 & 0 & 51 & 50.0 & 51 & 50.0 & 0 & 0 & 0 & 0 \\
\hline More than 28 services. . . & 12 & & .... & 4 & 33.3 & 8 & 66.7 & 0 & 0 & 0 & 0 & 0 & $\mathbf{0}$ \\
\hline 8 programs: & & & & & & & & & & & & & \\
\hline 8 services...... & 1 & & . & 0 & 0 & 0 & 0 & 0 & 0 & 1 & 100.0 & 0 & 0 \\
\hline $9-20$ services. .... & 76 & & ... & 0 & 0 & 0 & 0 & 53 & 69.7 & 23 & 30.3 & 0 & 0 \\
\hline 21-30 services . . . . . . . & 108 & & & 0 & 0 & 67 & 62.0 & 41 & 38.0 & 0 & 0 & 0 & 0 \\
\hline More than 30 services... & 38 & & .... & 29 & 76.3 & 9 & 23.7 & 0 & 0 & 0 & 0 & 0 & 0 \\
\hline 9 programs: & & & & & & & & & & & & & \\
\hline 9 services......... & 1 & 0 & 0 & 0 & 0 & 0 & 0 & 0 & 0 & 1 & 100.0 & 0 & 0 \\
\hline $10-25$ services. & 67 & 0 & 0 & 0 & 0 & 15 & 22.4 & 38 & 56.7 & 14 & 20.9 & 0 & 0 \\
\hline 26-35 services. . . . . . . . & 102 & 0 & 0 & 45 & 44.1 & 57 & 55.9 & 0 & 0 & 0 & 0 & 0 & 0 \\
\hline More than 35 services & 44 & 14 & 31.8 & 30 & 68.2 & 0 & 0 & 0 & 0 & 0 & 0 & 0 & 0 \\
\hline 10 programs: & & & & & & & & & & & & & \\
\hline 10 services...... & 2 & 0 & 0 & 0 & 0 & 0 & 0 & 0 & 0 & 2 & 100.0 & 0 & 0 \\
\hline 11-30 services.... & 53 & 0 & 0 & 0 & 0 & 29 & 54.7 & 13 & 24.5 & 11 & 20.8 & 0 & 0 \\
\hline 31-40 services......... & 46 & 3 & 6.5 & 41 & 89.1 & 2 & 4.3 & 0 & 0 & 0 & 0 & 0 & 0 \\
\hline More than $\mathbf{4 0}$ services. & 59 & 59 & 100.0 & 0 & 0 & 0 & 0 & 0 & 0 & 0 & 0 & 0 & 0 \\
\hline
\end{tabular}

Note: Each local health unit received 1 point for each of the services listed in table 8 that it provided. The total raw score for each unit was then converted to an index value according to the following formula: $\mathrm{I}=$ score of $40-48$ (high degree of involvement), II =32-39 (relatively high degree of involvement), $\mathrm{III}=24-31$ (above average involvement), $\mathrm{IV}=$ 16-23 (below average involvement), $\mathrm{V}=8-15$ (low involvement), $\mathrm{V} I=7$ or less (rare or minimum involvement). 
where another agency operated an information and referral service, a crippled children's program, a school health program, or a migrant health program.

Ten types of medical care programs thought to be commonly operated by local health units were identified, and the local health units surveyed were asked if they had any of these programs, what services were offered, how these services were provided, and what population groups were eligible. Tabulations of responses to these questions are shown in table 8. The most commonly available programs were tuberculosis control, 97 percent; venereal disease control, 85 percent; and child health programs, 84 percent. Cancer control programs were operated in only 42 percent of the units.

Most frequently, services were provided through the staff or facility of the local health unit, with the exception of in-hospital care and the services provided in crippled children's and cancer control programs.
The health units were asked what population groups were served under these programs. While many unusable responses were obtained, a general pattern emerged. Services such as screening, diagnosis, and health maintenance were usually provided to the general population or to specific age groups, while treatment services such as inhospital care, physicians' services, and drugs were usually provided only to the indigent. The notable exceptions to this pattern were the dental health programs, which served mainly indigent children, and the venereal disease control programs, in which treatment services were available to all persons with a positive diagnosis.

\section{Indexes of Involvement}

In order to obtain a general view of the involvement of each health unit in medical care, two indexes were developed which combined responses to questions concerning involvement in organizing medical care programs and in-

Table 10. Index of involvement in organizing medical care programs, by population of jurisdiction, type of health unit, employment status of health officer, and academic degrees of health officer

\begin{tabular}{|c|c|c|c|c|c|c|c|c|c|c|c|c|c|}
\hline \multirow{3}{*}{ Item } & \multirow{3}{*}{$\begin{array}{c}\text { Total } \\
\text { health } \\
\text { units }\end{array}$} & \multicolumn{12}{|c|}{ Index } \\
\hline & & \multicolumn{2}{|c|}{$\mathbf{I}$} & \multicolumn{2}{|c|}{ II } & \multicolumn{2}{|c|}{ III } & \multicolumn{2}{|c|}{ IV } & \multicolumn{2}{|c|}{$\mathbf{V}$} & \multicolumn{2}{|c|}{ VI } \\
\hline & & $\underset{\text { ber }}{\text { Num- }}$ & $\begin{array}{l}\text { Per- } \\
\text { cent }\end{array}$ & $\underset{\text { ber }}{\text { Num- }}$ & $\begin{array}{l}\text { Per- } \\
\text { cent }\end{array}$ & $\underset{\text { ber }}{\text { Num- }}$ & $\begin{array}{l}\text { Per- } \\
\text { cent }\end{array}$ & $\underset{\text { ber }}{\text { Num- }}$ & $\begin{array}{l}\text { Per- } \\
\text { cent }\end{array}$ & $\underset{\text { ber }}{\text { Num- }}$ & $\begin{array}{l}\text { Per- } \\
\text { cent }\end{array}$ & $\underset{\text { ber }}{\text { Num- }}$ & $\begin{array}{l}\text { Per- } \\
\text { cent }\end{array}$ \\
\hline Total & 1,323 & 76 & 5.7 & 149 & 11.3 & 248 & 18.7 & 329 & 24.9 & 320 & 24.2 & 201 & 15.2 \\
\hline \multicolumn{14}{|l|}{ Population of jurisdiction: } \\
\hline Under 15,000 & 170 & 13 & 7.6 & 11 & 6.5 & 18 & 10.6 & 36 & 21.2 & 54 & 31.8 & 38 & 22.4 \\
\hline $15,000-24,999$ & 202 & 13 & 6.4 & 22 & 10.9 & 29 & 14.4 & 44 & 21.8 & 58 & 28.7 & 36 & 17.8 \\
\hline $25,000-44,999$ & 266 & 15 & 5.6 & 33 & 12.4 & 58 & 21.8 & 57 & 21.4 & 56 & 21.1 & 47 & 17.7 \\
\hline $45,000-74,999 \ldots$ & 244 & 13 & 5.3 & 25 & 10.2 & 61 & 25.0 & 52 & 21.3 & 53 & 21.7 & 40 & 16.4 \\
\hline $75,000-199,999$ & 225 & 13 & 5.8 & 34 & 15.1 & 39 & 17.3 & 66 & 29.3 & 49 & 21.8 & 24 & 10.7 \\
\hline 200,000 and over.......... & 164 & 9 & 5.5 & 17 & 10.4 & 35 & 21.3 & 55 & 33.5 & 38 & 23.2 & 10 & 6. 1 \\
\hline Unknown. & 52 & 0 & 0 & 7 & 13.5 & 8 & 15.4 & 19 & 36.5 & 12 & 23.1 & 6 & 11.5 \\
\hline \multicolumn{14}{|l|}{ Type of health unit: } \\
\hline County units..... & 687 & 31 & 4.5 & 76 & 11.1 & 143 & 20.8 & 204 & 29.7 & 164 & 23.9 & 69 & 10.0 \\
\hline City units.................. & 247 & 9 & 3.6 & 20 & 8.1 & 23 & 9.3 & 30 & 12.1 & 84 & 34.0 & 81 & 32.8 \\
\hline City-county units.......... & 74 & 3 & 4.1 & 2 & 2.7 & 9 & 12.2 & 27 & 36.5 & 22 & 29.7 & 11 & 14.9 \\
\hline State districts..... & 123 & 19 & 15.4 & 24 & 19.5 & 23 & 18. 7 & 27 & 22.0 & 15 & 12.2 & 15 & 12.2 \\
\hline Local districts & 192 & 14 & 7.3 & 27 & 14.1 & 50 & 26.0 & 41 & 21.4 & 35 & 18.2 & 25 & 13.0 \\
\hline \multicolumn{14}{|l|}{ Employment status of health officer: } \\
\hline Full-time officers & 762 & 53 & 7.0 & 108 & 14.2 & 144 & 18.9 & 198 & 26.0 & 171 & 22.4 & 88 & 11.5 \\
\hline Part-time officers. & 441 & 16 & 3.6 & 33 & 7.5 & 78 & 17.7 & 100 & 22.7 & 125 & 28.3 & 89 & 20.2 \\
\hline No health officer. & 95 & 7 & 7.4 & 5 & 5.3 & 22 & 23.2 & 27 & 28.4 & 17 & 17.9 & 17 & 17.9 \\
\hline Unknown status. & 25 & $\mathbf{0}$ & $\mathbf{0}$ & 3 & 12.0 & 4 & 16.0 & 4 & 16.0 & 7 & 28.0 & 7 & 28.0 \\
\hline \multicolumn{14}{|l|}{ Academic degrees of health officer: } \\
\hline M.D.'s with public health degree. & 415 & 30 & 7.2 & 61 & 14.7 & 88 & 21.2 & 114 & 27.5 & 94 & 22.7 & 28 & 6.7 \\
\hline M.D.'s without public health degree........ & 645 & 35 & 5.4 & 74 & 11.5 & 123 & 19.1 & 168 & 26.0 & 147 & 22.8 & 98 & 15.2 \\
\hline Non-M.D.'s with public health degree...... & 26 & $\mathbf{0}$ & 0 & 4 & 15.4 & 0 & 0 & 5 & 19.2 & 4 & 15.4 & 13 & 50.0 \\
\hline Non-M.D.'s without public health degree... & 107 & 3 & 2.8 & 4 & 3.7 & 8 & 7.5 & 11 & 10.3 & 41 & 38.3 & 40 & 37.4 \\
\hline No health officer. & 95 & 7 & 7.3 & 5 & b. 3 & 22 & 23.2 & 28 & 29.5 & 17 & 17.9 & 16 & 16.8 \\
\hline 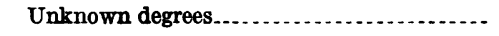 & 35 & 1 & 2.9 & 1 & 2.9 & 7 & 20.0 & 3 & 8.6 & 17 & 48.6 & 6 & 17.1 \\
\hline
\end{tabular}


volvement in community health. The remainder of this paper focuses on these indexes.

Organizing medical care programs. The index of involvement in organizing medical care programs was designed to classify each health unit according to its involvement in directly providing 10 selected programs in terms of the range and depth of services offered in each (table 9). The index does not fully reflect the depth of the programs since it does not take into account the size of the population served.

A 6-point scale ranging from I, for units with a high degree of involvement, to VI, for units with a minimal degree of involvement, was used. Less than 6 percent of all health units attained an index value of I, while 15 percent rated an index value of VI. Almost 65 percent of the units provided less than 24 of a possible 48 services used to score the index. Only 20 units reported they offered none of the 10 programs in their jurisdictions.

The components of this index are analyzed in table 9 by the number of programs and the number of services offered. Seventeen percent of the units fell in the two highest indexes, and these were units which offered not only a broad range of programs but also a considerable depth of services. All of these units offered at least seven programs out of a possible 10 and at least 28 services out of a possible 48 . The majority had nine programs and offered at least 35 services. Of the 201 units which received the lowest index value, only one offered as many as seven programs, and this unit offered only one service in each of those seven programs. The majority of units with low indexes offered a very narrow range of services within a few programs.

Approximately 70 percent of the units fell in the midrange from index III to index V. Units obtaining an index score of III offered between 6 and 10 programs and had a fair depth of services within these programs, whereas those units with indexes of IV and V offered from 3 to 10 programs but tended to lack depth in the number of services offered in these programs.

As might be expected, units with higher per capita public health expenditures, serving larger populations, and having larger budgets more frequently tend to obtain the higher index values (table 10, fig. 1).

Figure 1. Percentage distribution of index of involvement of local health units in organizing medical care programs, by per capita public health expenditures

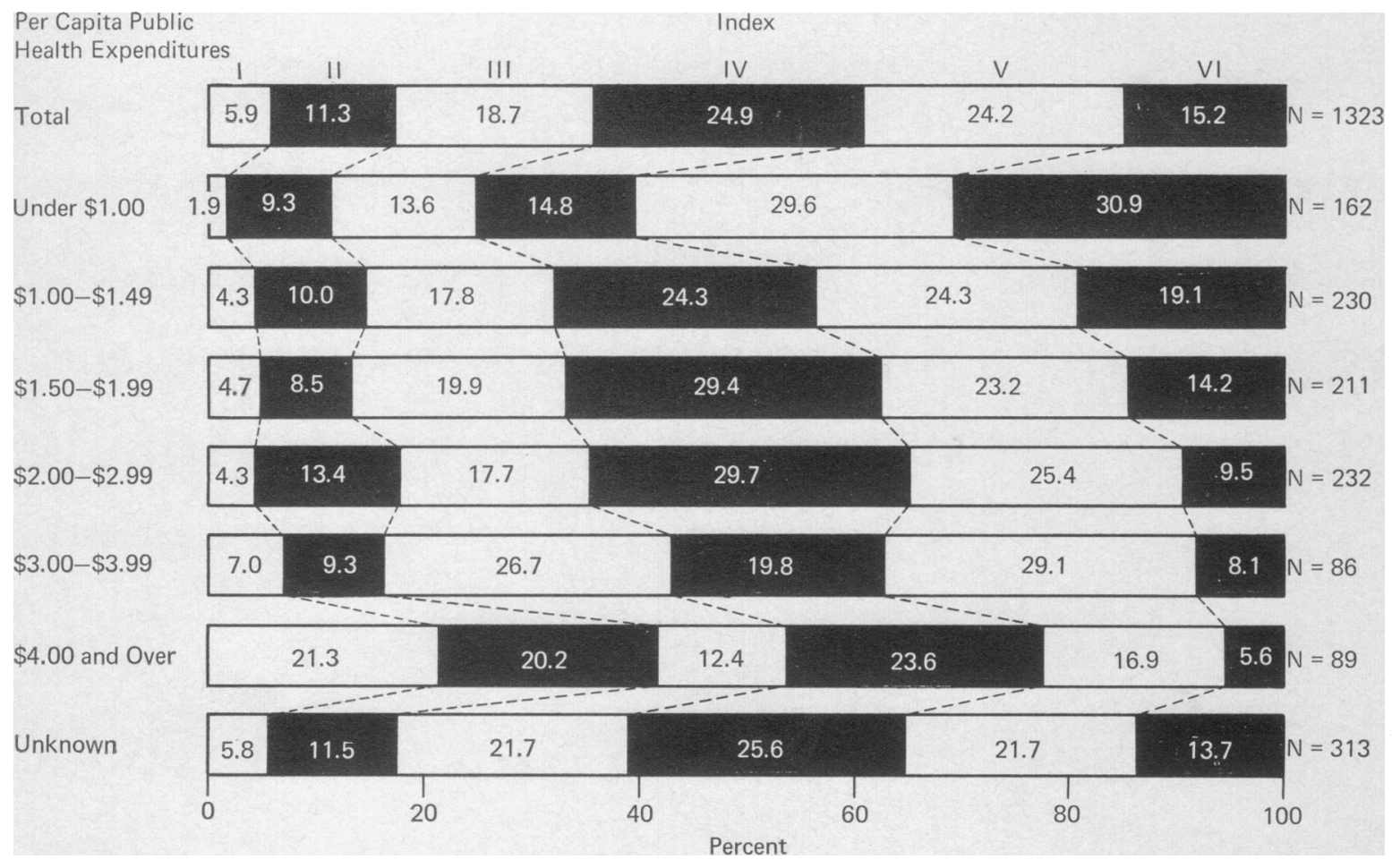


Table 11. Raw scores received by 1,323 local health units on components of index of involvement in community health

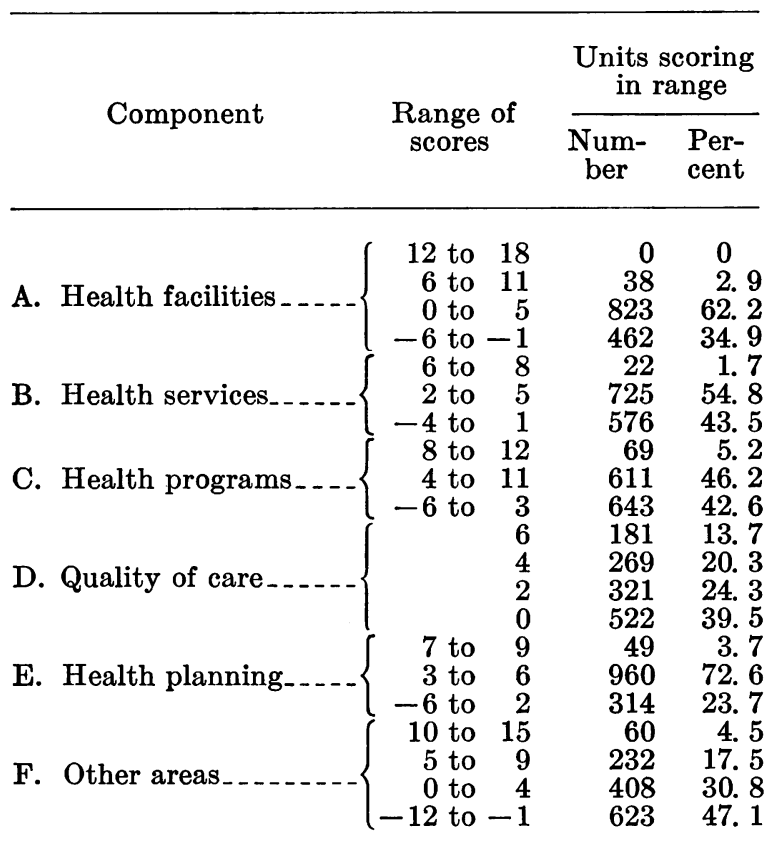

The employment status and qualifications of the health officer have a direct relationship to the involvement of the unit in organizing medical care programs (table 10). While 40 percent of the units with a full-time health officer and 36 percent of those with no health officer had index values of I to III, only 29 percent of the units which had part-time health officers had index values in this range. Units in which the health officer was a physician with a public health degree had a greater degree of involvement than the units in which the health officer was a physician without a public health degree. Moreover, units with health officers who were not physicians, with or without a public health degree, were considerably less involved in organizing medical care programs than the units with physician health officers or the units without health officers.

Community health. The index of involvement in community health, in contrast to the previous index, was designed to measure the scope of each local health unit's relationships with other community health agencies and programs and its general involvement in community health affairs. The index score for each health unit was obtained by summing the raw scores on six component subparts and converting this raw score into an index value.

In subpart $A$, the health units were assigned 1 point for each of the following responsibilities assumed for each of the six types of health facilities listed in table 5 : operates facility, licenses or inspects facility or both, and has other responsibility. In addition, 1 point was subtracted where facilities of a particular type were available in the jurisdiction, but the health unit reported no responsibilities. As shown in table 11 , only 3 percent of the health units had a score greater than 6 and 35 percent had negative scores, indicating that few health units have a wide range of responsibilities with respect to health facilities in their jurisdictions.

Subpart B of the index reflects the assistance that health units give to information and referral, home nursing, homemaker, and home health services. A unit was assigned 1 point for each service that it provided directly and 1 point for each service which it assisted another agency in operating. However, if the unit provided no assistance to another agency operating such a service, a minus 1 was added to its score. More than 40 percent of the health units had scores of 1 or less on this subpart, suggesting a considerable lack of interagency relationships.

Subpart C relates to the health unit's activities with respect to medical assistance, crippled children's, school health, vocational rehabilitation, migrant health, and mental health programs. The index was scored in the same manner as subpart B. Only 5 percent of the health units had scores ranging from 8 to 12 , indicat-

\section{Table 12. Scoring of 1,323 local health units on index of involvement in community} health

\begin{tabular}{|c|c|c|c|}
\hline \multirow[t]{2}{*}{ Index } & \multirow{2}{*}{$\begin{array}{l}\text { Range of } \\
\text { total raw } \\
\text { scores }\end{array}$} & \multicolumn{2}{|c|}{$\begin{array}{l}\text { Units scoring in } \\
\text { range }\end{array}$} \\
\hline & & Number & Percent \\
\hline Total__._. & -34 to 68 & 1,323 & 100. 0 \\
\hline 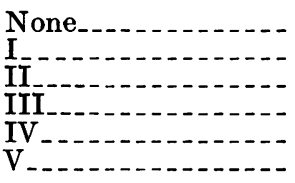 & $\begin{array}{r}54 \text { to } 68 \\
39 \text { to } 53 \\
24 \text { to } 38 \\
9 \text { to } 23 \\
-6 \text { to } 8 \\
-34 \text { to }-7\end{array}$ & $\begin{array}{r}0 \\
9 \\
136 \\
586 \\
532 \\
60\end{array}$ & \begin{tabular}{r}
\multicolumn{1}{c}{0} \\
10.7 \\
44.3 \\
40.2 \\
4.5
\end{tabular} \\
\hline
\end{tabular}


ing a high level of cooperation with or operation of such programs. On the other hand, more than 40 percent of the health units had a score of 3 or less, indicating little activity with respect to these programs.

Subpart D scores were given for activities undertaken by the local health unit in the past 5 years to improve professional skills, quality of services, or administrative procedures of other public or private health agencies. Two points were awarded for each of these three categories in which the unit reported at least one activity. Almost 40 percent of the health units reported undertaking no such activities in the past 5 years. Fourteen percent of the health units reported at least one activity in all three categories.

Subpart E of the index reflects the participation of the local health unit in the activities of the local health and welfare council, the hospital planning agency, and the regional or State health council. The local health unit's relationship with each of these health planning agencies was scored as follows: 3 points if the health officer or his representative assumed a leadership role, 2 points if he participated regularly, 1 point if he occasionally participated, minus 2 points if he did not participate, and 1 point if no agency was active in the health unit's jurisdiction. Almost 25 percent of the health units had a raw score of 2 or less on this subpart, indicating minimal or no participation in such health planning activities. Only 4 percent of the health units could be termed "very active participants" in community health planning. The vast majority of the health units fell between these two extremes and could be considered to be participating more or less regularly in such activities.

The last component of this index, subpart F, summarizes the relationships between the local health unit and the local medical and dental societies, local hospitals, and medical and public health schools in the area. Also included were scores for encouraging other agencies to undertake major activities related to personal health services and for general coordination of medical care activities. Health units were assigned negative points if they failed to take advantage of opportunities to establish such rela-

Figure 2. Percentage distribution of index of involvement of local health units in community health, by index of involvement in organizing medical care programs

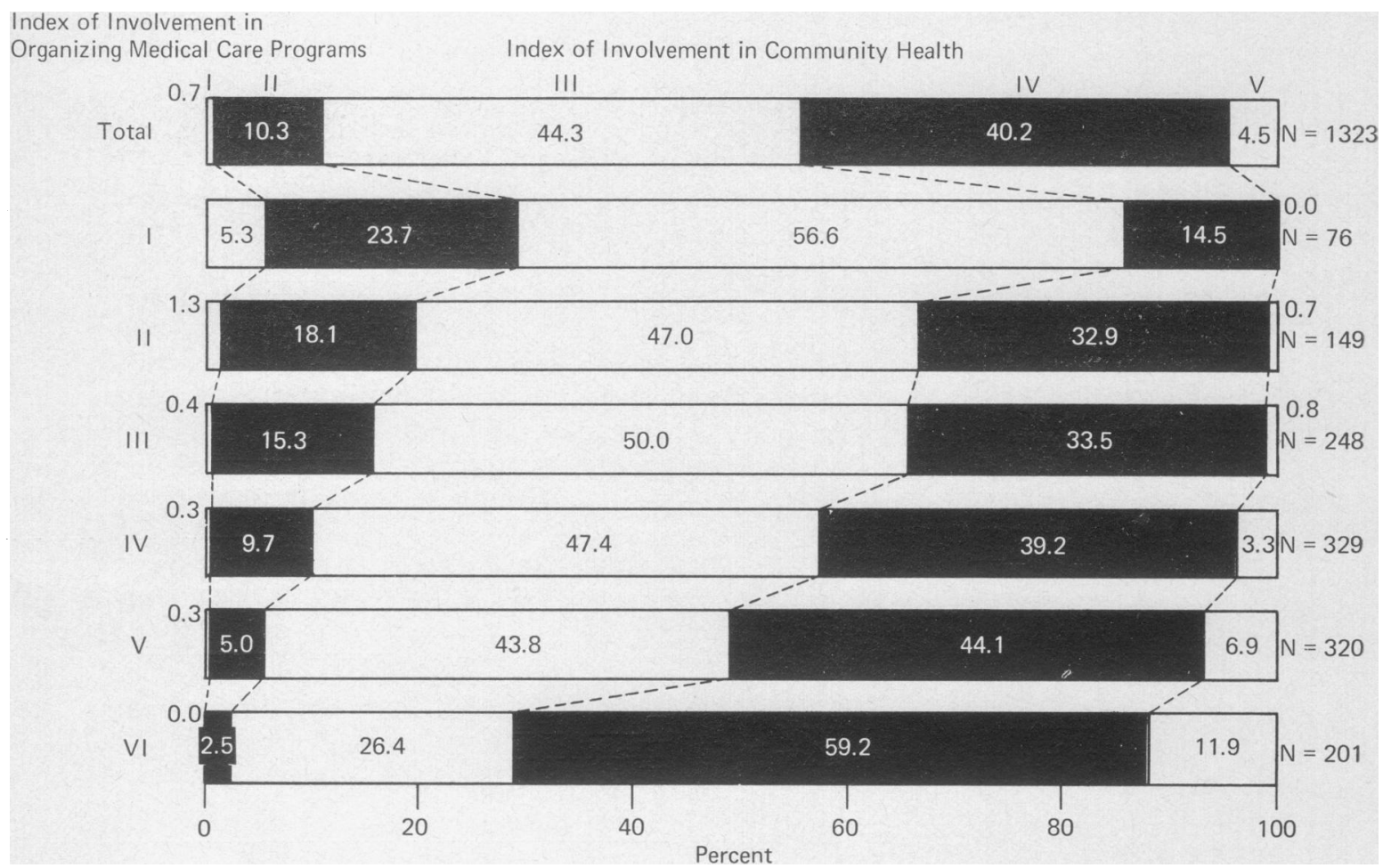


tionships and activities. Almost half of the health units had negative scores on this subpart, while less than 5 percent of the health units had scores indicating a high degree of involvement.

When the raw scores on each of the subparts were totaled for each health unit and converted into an index score, none of the health units scored in the highest index (table 12). Thus, a 5 -point scale was used instead of the 6-point scale. Even with this adjustment only 11 percent of the health units attained index scores of I and II, which reflect a relatively high degree of

Table 13. Index of involvement in community health, by employment status and academic degrees of health officer

\begin{tabular}{|c|c|c|c|c|c|c|c|c|c|c|c|}
\hline \multirow{3}{*}{ Item } & \multirow{3}{*}{ Total } & \multicolumn{10}{|c|}{ Index } \\
\hline & & \multicolumn{2}{|r|}{ I } & \multicolumn{2}{|c|}{ II } & \multicolumn{2}{|c|}{ III } & \multicolumn{2}{|c|}{ IV } & \multicolumn{2}{|c|}{$\mathrm{V}$} \\
\hline & & Number & Percent & Number & Percent & Number & Percent & Number & Percent & Number & Percent \\
\hline Total .... & 1,323 & 9 & 0.7 & 136 & 10.3 & 586 & 44.3 & 532 & 40.2 & 60 & 4.5 \\
\hline \multicolumn{12}{|l|}{ Employment status of health officer: } \\
\hline Full-time health officers................. & 762 & 8 & 1.0 & 111 & 14.6 & 353 & 46.3 & 258 & 33.9 & 32 & 4.2 \\
\hline Part-time health officers................ & 441 & 1 & .2 & 22 & 5.0 & 188 & 42.6 & 212 & 48.1 & 18 & 4.1 \\
\hline No health officer & 95 & 0 & 0 & 2 & 2.1 & 36 & 37.9 & 49 & 51.6 & 8 & 8.4 \\
\hline Unknown status & 25 & $\mathbf{0}$ & $\mathbf{0}$ & 1 & 4.0 & 9 & 36.0 & 13 & 52.0 & 2 & 8.0 \\
\hline \multicolumn{12}{|l|}{ Academic degrees of health officer: } \\
\hline M.D.'s with public health degree...... & 415 & 6 & 1.4 & 72 & 17.3 & 193 & 46.5 & 135 & 32.5 & 9 & 2.2 \\
\hline $\begin{array}{l}\text { M.D.'s without public health degree.- } \\
\text { Non-M.D's with public health de- }\end{array}$ & 645 & 3 & .5 & 55 & 8.5 & 295 & 45.7 & 265 & 41.1 & 27 & 4.2 \\
\hline gree & 26 & 0 & 0 & 3 & 11.5 & 13 & 50.0 & 9 & 34.6 & 1 & 3.8 \\
\hline $\begin{array}{l}\text { Non-M.D.'s without public health de- } \\
\text { gree-. }\end{array}$ & 107 & $\mathbf{0}$ & 0 & 4 & 3.7 & 36 & 33.6 & 54 & 50.5 & 13 & 12.1 \\
\hline No health officer... & 95 & 0 & 0 & 2 & 2.1 & 36 & 37.9 & 49 & 51.6 & 8 & 8.4 \\
\hline Degrees unknown..... & 35 & $\mathbf{0}$ & $\mathbf{0}$ & 0 & $\mathbf{0}$ & 13 & 37.1 & 20 & 57.1 & 2 & 5.7 \\
\hline
\end{tabular}

Figure 3. Percentage distribution of index of involvement of local health units in community health, by per capita public health expenditures

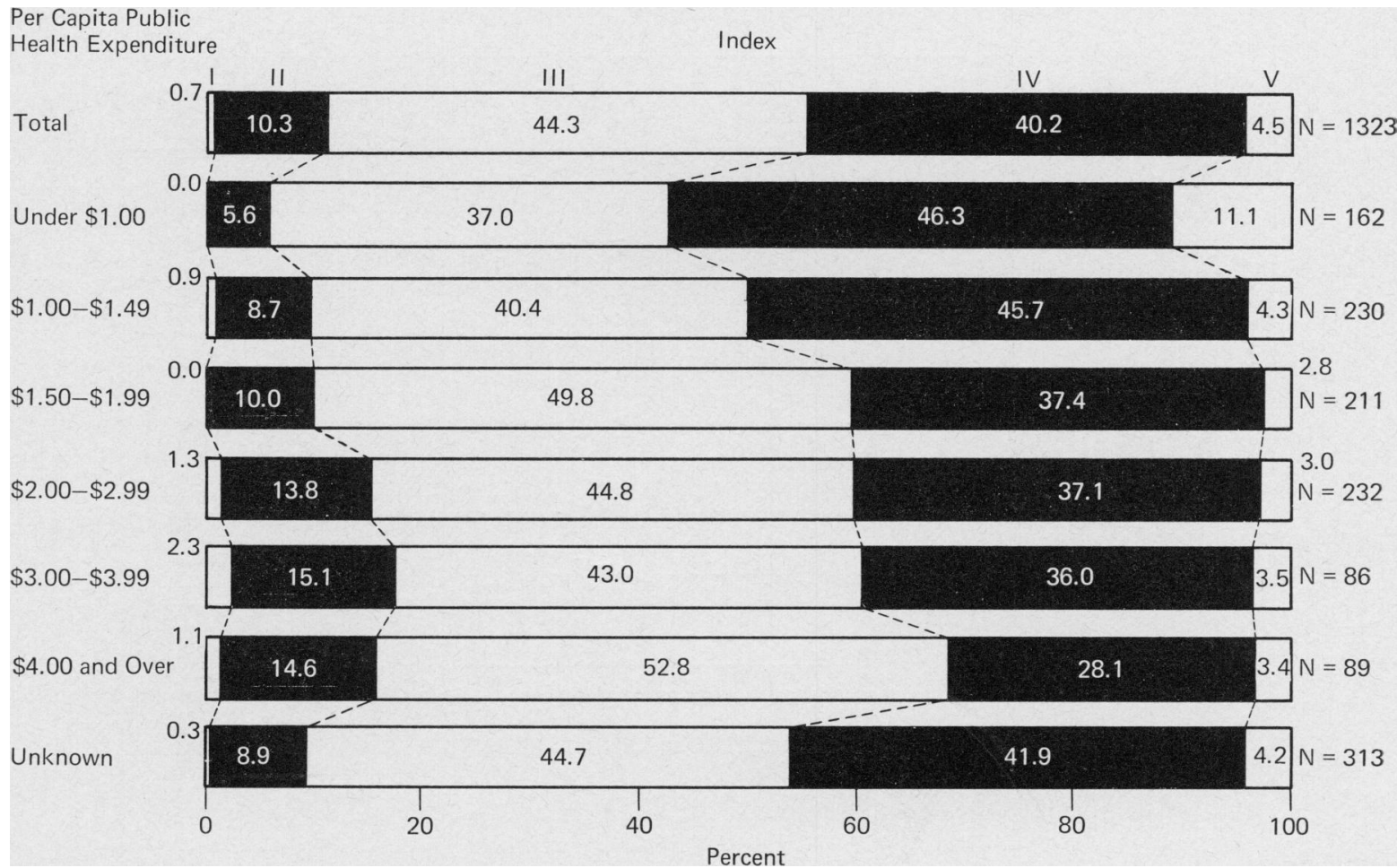


involvement in community health affairs. On the other hand, 45 percent of the health units received low index values of $I V$ and $V$.

The index is not intended to describe the quality of a unit's relationships in the community. A unit, for example, might have one or two important planning and coordination activities, but score low in the index because it did not undertake other activities. On the other hand, a unit might score high on the index by conducting many interagency activities, but the index does not reflect how effective such relationships actually are. Recognizing these limitations, we believe the index, with its subparts, has value in describing the scope of involvement of local health units in areas of community health presumed to be important in a public health and medical care program.

The degree of association between the index of involvement in community health and the index of involvement in organizing medical care programs is indicated in figure 2. As shown, a substantial proportion of the units with high indexes of involvement in organizing medical care programs also scored well on the index of
Table 14. Percent of local health units providing specified programs in 1947 and 1966

\begin{tabular}{|c|c|c|}
\hline \multirow[t]{2}{*}{ Program } & \multicolumn{2}{|c|}{$\begin{array}{l}\text { Percent of } \\
\text { health units }\end{array}$} \\
\hline & $1947^{1}$ & $1966^{2}$ \\
\hline Venereal disease_ & 88 & 85 \\
\hline - & 86 & 97 \\
\hline Crippled children & 62 & 72 \\
\hline Child health & 78 & 84 \\
\hline Dental health - & 46 & 66 \\
\hline Maternal health: & & \\
\hline Prenatal clinics & 48 & 62 \\
\hline Delivery 3 & 11 & 20 \\
\hline - . - & 28 & 42 \\
\hline Mental hygiene & 20 & 55 \\
\hline Cardiac......... & 21 & 57 \\
\hline
\end{tabular}
(3)

1 Based on 690 reporting full-time local health units 2 Based on 1,323 reporting local health units.

8 In-hospital delivery only in 1966.

involvement in community health, whereas the units with low involvement in organizing medical care programs also tended to have low involvement in community health. The coefficient of correlation between these two indexes is 0.33 .

Figure 4. Percentage distribution of index of involvement of local health units in community health, by population of jurisdiction

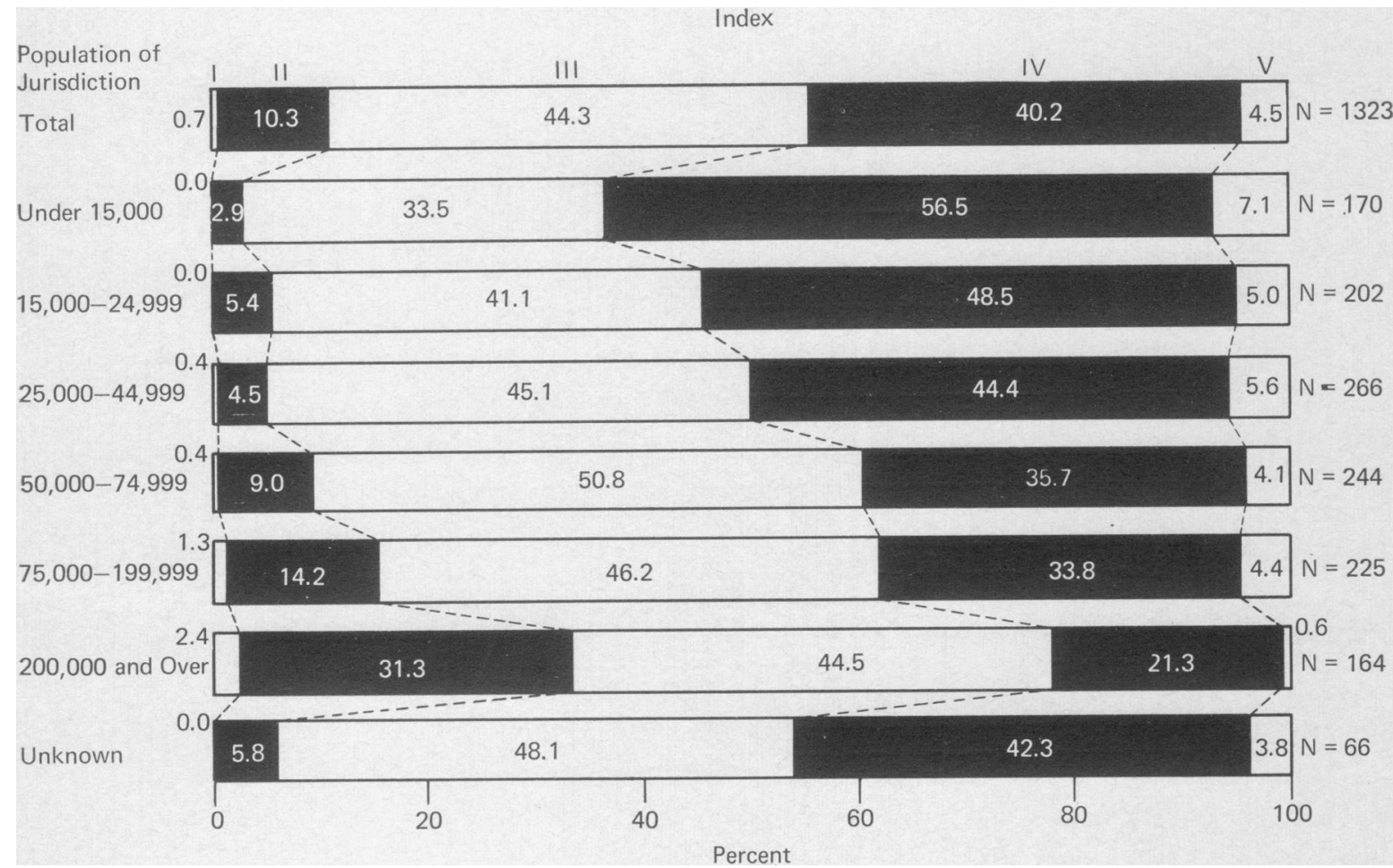


Thus, local health units which are deeply involved in providing medical care services to the populations they serve also tend to have numerous relationships with other agencies and organizations in their jurisdictions.

As might be expected, therefore, the same factors exerting a positive influence on the index of involvement in organizing medical care programs have a similar relation to the index of involvement in community health. This is demonstrated in table 13 and figures 3 and 4. However, comparison of figures 1 and 2 suggests that per capita public health expenditures have less influence on the community health involvement. On the other hand, the size of the population of the jurisdiction served by the health unit has a considerably greater effect on involvement in community health (fig. 4).

\section{Discussion}

This study will provide a wealth of data previously unavailable. A full report on the data gathered, with more complete analyses than presented here, is forthcoming.

While the Terris-Kramer study of full-time local health units in the late 1940's (3) is not strictly comparable with this study, due to differences in definitions, sampling, and the questions asked, the comparisons which can be made point to an increase in involvement in providing medical care services by local health units since that time. A comparison of all local health units reporting in 1966 with the 690 full-time health units responding in 1947 (table 14) reveals that of the 10 programs for which comparisons can be made, nine were provided more frequently in 1966. The only program less available in 1966 was venereal disease control, which was provided by 3 percent fewer health units.

Since much of this presentation was directed to the two indexes developed for this study, comment is due on the implications of our find- ings. The need for the direct provision of personal health services by a local health unit depends on the needs and special problems of the population it serves, which cannot be evaluated in a survey of this type. However, as the 1963 policy statement of the American Public Health Association suggested (2) :

The local health department should play a leadership role in community-wide organization and planning of health resources. It should also promote the full use by physicians, hospitals, and others of all community resources which provide educative, preventive, diagnostic, therapeutic and rehabilitative services. It should develop effective techniques that are easily applied to large numbers of people. When it has done this, it should mobilize community resources to apply them.

The findings reported here suggest that many health units have not yet achieved this goal. Certainly it is a goal toward which they should be aiming, and one may assume that when the full impact of Medicare, Medicaid, and comprehensive health services under P.L. 89-749 is felt, this picture will change. It will be incumbent upon the Public Health Service to reassess the medical care activities of local health departments again in the future.

\section{REFERENCES}

(1) The local health department-services and responsibilities: An official statement of the American Public Health Association. (Adopted Nov. 1, 1950.) Amer J Public Health 41 : 302-307, March 1951.

(2) The local health department-services and responsibilities : An official statement of the American Public Health Association, (Adopted Nov. 10, 1963.) Amer J Public Health 54 : 1, January 1964.

(3) Terris, M., and Kramer, N. A.: Medical care activities of full-time health departments. Amer J Public Health 39: 1129-1135, September 1949.

(4) U.S. Public Health Service: Directory of local health units, 1966. PHS Publication No. 118, U.S. Government Printing Office, Washington, D.C., 1966. 


\section{Program Notes}

\section{Cartoon Booklet on Drugs}

The Pennsylvania Health Council has published a booklet with cartoon type illustrations entitled "Drugs and People-about unhealthy dependence." Originally prepared and published in Canada by the Alcoholism and Drug Addiction Research Foundation in Toronto, the booklet is available to health departments and other agencies at 15 cents a copy

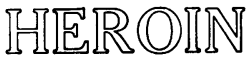

\section{IS A MIXTURE \\ OF MORPHINE \\ AND ACETIC ACID \\ WHICH WAS \\ DISCOVERED IN \\ 1898. AT FIRST CONSIDERED NON- ADDICTING AND A SAFE SUBSTITUTE FOR MORPHINE, IT IS NOW RECOGNIZED AS EVEN MORE ADDICTING.}

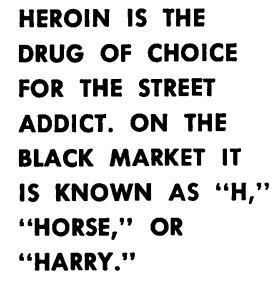

from the Pennsylvania Health Council, Inc., 105 North Front Street, Harrisburg, Pa. 17101.

"Drugs and People" briefly touches on and illustrates the use of and addiction to alcohol, barbiturates, tranquilizers, opium, morphine, heroin, amphetamine, cocaine, marihuana, LSD, and nicotine. The page on heroin is reproduced in the illustration.

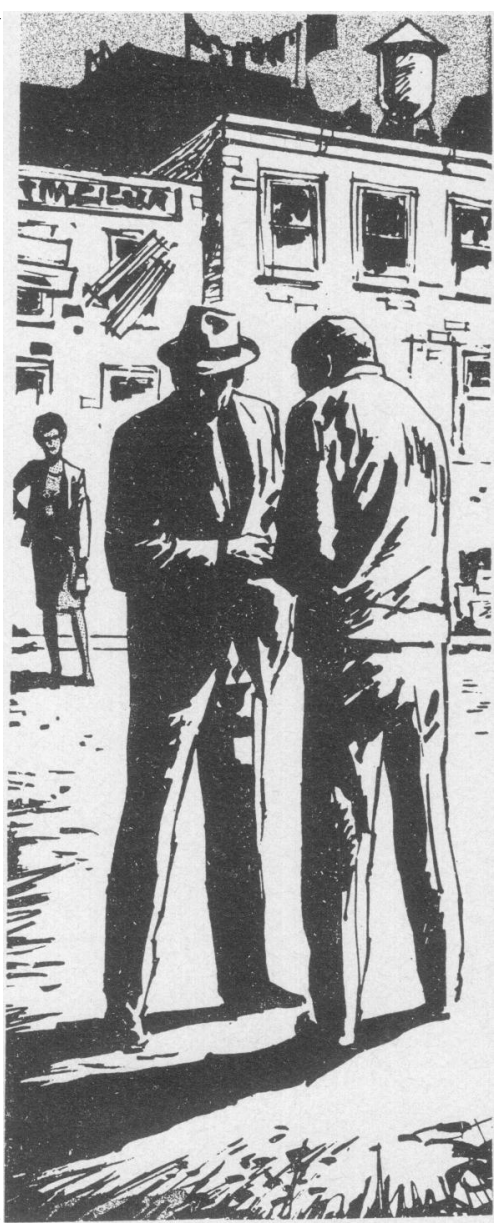

oline brands, surveyed about $\mathbf{1 0 , 0 0 0}$ gas stations in New York State during the summer of 1968 to compile a report on disposal of old crankcase oil. Dr. Hollis S. Ingraham termed the survey important. "If oil is dumped into the sewers," he pointed out, "it causes water pollution; if it is burned, it causes air pollution; and if it is poured on the ground, it can pollute subsurface ground waters."

Gas station operators who in the past sold used oil to reprocessors now have to pay to get it taken away. According to industry sources, the change results from the lifting of a Federal excise tax on new oil and a Federal ruling that reprocessed oil must be labeled as such.

\section{Attack on Hay Fever}

The New York State Department of Health provides a daily ragweed pollen count from July 29 through September 27. Volunteers working throughout the State make it possible to issue reports of the counts, based on 24-hour tests, by noon each day during this period. Readings of 7 or above indicate a "hay fever day." Readings of 25 or higher signify high contamination.

Forty-six communities participated in the hay fever control program in 1967, and the department expected at least as many to participate in 1968.

More than 1 million New York State residents suffer from hay fever, according to Dr. Hollis S. Ingraham, the State health commissioner. Ragweed blossoms and begins to bother these people in late July. It should therefore be sprayed, cut, or destroyed before that time, Ingraham pointed out.

\section{Toll-Free Cancer Consultations}

Roswell Park Memorial Institute, the cancer research facility operated by the New York State Department of Health, provides a tollfree 24-hour telephone consultation service to licensed physicians and dentists practicing in New York State.

Items for this page: Health departments, health agencies, and others are invited to share their program successes with others by contributing items for brief mention on this page. Flag them for "Program Notes" and address as indicated in masthead. 